\title{
Airway Management and Assessment of Dyspnea in Emergency Department Patients with Acute Heart Failure
}

\author{
Peter S. Pang $\cdot$ Masood Zaman
}

Published online: 8 March 2013

(c) Springer Science+Business Media New York 2013

\begin{abstract}
Shortness of breath is the commonest symptom in patients with acute heart failure. Ensuring adequate oxygenation and ventilation as well as symptomatic relief are key goals of early emergency department management. In this focused review, we describe how to assess dyspnea in clinical practice and how to treat acute heart failure patients to relieve dyspnea, with initial discussion on airway and breathing management for patients who present in extremis.
\end{abstract}

Keywords Acute heart failure - Dyspnea - Airway . Breathing · Emergency department · Noninvasive ventilation

\section{Introduction}

Acute heart failure (AHF) patients present to the emergency department (ED) primarily because of the sensation of breathlessness, or dyspnea $[1 \bullet \bullet, 2,3]$. This sensation can be so severe that some patients with advanced heart failure would consider trading survival time for symptom relief [4]. Alleviating dyspnea is a key goal of therapy.

Traditional AHF management with intravenous loop diuretics, nitrovasodilators, morphine, and oxygen reduces breathlessness significantly for most patients [1••], although a sizable minority continue to have symptoms for up to $48 \mathrm{~h}$ after initial management [3]. This suggests that some patients require treatment beyond traditional management. In addition, other patients require additional interventions

P. S. Pang $(\varangle) \cdot$ M. Zaman

Department of Emergency Medicine, Northwestern Feinberg

School of Medicine, Chicago, IL 60521, USA

e-mail: ppang@northwestern.edu because of the severity of their respiratory distress. Noninvasive positive-pressure ventilation and, in rare circumstances, endotracheal intubation may be necessary to ensure sufficient oxygenation and ventilation, reduce the work of breathing, and further reduce the severity of symptoms. How to assess dyspnea from a clinician's perspective as well as when to consider noninvasive positive-pressure ventilation or endotracheal intubation will be discussed in this report.

\section{Assessment of Dyspnea in Clinical Practice}

Presently, a universally accepted and validated patientreported outcome instrument to assess dyspnea in the AHF clinical setting does not exist [2, 5•]. Clinical trials commonly use a Likert or visual analog scale to assess dyspnea [6-9], although these are not routinely used in clinical practice. This may be because physician assessment of dyspnea as opposed to the patient's subjective self-report more strongly influences initial management. However, such an approach reflects a potential shortcoming of current management; given the subjective nature of dyspnea and its role in driving hospital presentation for AHF, ensuring its relief from the patient perspective is critical $[4,10]$. Retrospective analysis suggests an association between severity of dyspnea and worse outcomes, such as increased length of stay, less relief from congestion, and increased mortality [3, 11]. Ultimately, use of a particular instrument is less important than ascertainment of the degree and severity of dyspnea from the patient's perspective.

After initial stabilization, all AHF patients should be asked about the extent and severity of dyspnea and its impact on their daily living. Practically, asking about usual daily activities (i.e., walking to work, up and down stairs, across the room, etc.) and comparing current activity levels 
with the level of activity prior to decompensation may provide a reference point for patients. A patient's ability to sleep comfortably (i.e., absence of paroxysmal nocturnal dyspnea and orthopnea) may be another clue to the degree and severity of patient discomfort. For example, could the patient lie flat in the past, but is now sleeping on several pillows or in a reclining chair? As a general rule, discordance between physician assessment and patient-reported dyspnea should prompt further investigation. For some patients, compliance with medications and diet, education on the progression of heart failure, and/or in-depth knowledge of patient preferences may be sufficient. For other patients, occult causes of dyspnea (e.g., pulmonary embolism) may have been overlooked.

\section{Management of the Airway in AHF}

The need for endotracheal intubation in AHF patients is relatively uncommon. Nevertheless, decisive management is occasionally necessary, driven primarily by clinical judgment. Although adjunctive assessments of respiratory status such as arterial blood gas measurement, oxygen saturation, respiratory rate, and use of accessory muscles may be helpful, the clinician should be careful to avoid overreliance on such measures. Anticipation of the clinical course is a key decision point in airway management, allowing a controlled urgent intubation versus an emergent one [12].

For most patients, time will allow careful assessment of the airway and breathing, as well as other clinical, historical, and physical examination features that will influence decision-making. For those with mild to moderate distress, respiratory rate, level of oxygen saturation, and extent of the ability to use accessory muscles will all be factors influencing the clinical decision to begin oxygen therapy. The threshold to start oxygen therapy should be relatively low. For patients with severe respiratory distress, oxygen therapy should be applied immediately and consideration of definitive airway management occurs in parallel with rapid pharmacological management. If immediate endotracheal intubation is unnecessary, noninvasive ventilation (NIV) should be strongly considered and is recommended [13].

\section{Endotracheal Intubation with Rapid-Sequence Intubation}

Rapid-sequence intubation (RSI) is recommended to definitively secure the airway in the ED and is both safe and effective [12]. RSI involves near-simultaneous administration of a rapid-acting sedative along with a short-acting paralytic without bag-valve-mask ventilation. Preoxygenation to avoid hypoxia for as long as possible during apnea is critical. For pulmonary edema patients, the time to hypoxia will be much shorter than for healthy adults [12]. More recent data demonstrate the value of a high-flow nasal cannula during RSI to delay onset of hypoxia and we recommend its application [14]. If patients are hypoxic to begin with, "bagging" the patient prior to RSI may be necessary. The risk of aspiration due to vomiting from bag-valve-mask oxygenation and ventilation in patients with a potentially full stomach versus the risk of hypoxia needs to be carefully weighed.

\section{Specific RSI Drugs}

Rapidly acting sedative agents are used to achieve unconsciousness in RSI. Etomidate (induction dose of $0.3 \mathrm{mg} / \mathrm{kg}$ intravenously) is commonly used because of its rapid onset, brief duration of action, and lower risk of hypotension when compared with other induction agents. Benzodiazepines may also be used; however, caution is warranted. Midazolam (induction dose $0.3 \mathrm{mg} / \mathrm{kg}$ intravenously) has some negative chronotropic effects in addition to lowering blood pressure $[12,15]$. As a general rule, etomidate is the recommended induction agent of choice.

Immediately following administration of the sedative agent, a paralytic agent is given to improve intubation success through complete relaxation of the musculature. Although the "brutane" method of intubation or sedative alone approach avoids apnea, in general, it is an inferior approach compared with RSI in regard to intubation success in the emergent patient [12].

Succinylcholine is a commonly used paralytic agent (dose $1.5-2 \mathrm{mg} / \mathrm{kg}$ intravenously). Its brief half-life, fast onset, and few contraindications are some of its appealing properties. In addition to its depolarizing effects at the motor end plate, succinylcholine has some negative chronotropic activity owing to its stimulation of muscarinic receptors [12]. This effect is not common, but bradycardia may manifest itself with further doses of succinylcholine. If clinically indicated, atropine may be given for treatment of bradycardia. When in doubt regarding weight-based dosing, clinicians should err on the side of a higher dose.

For patients in whom treatment with succinylcholine is contraindicated, rocuronium, a nondepolarizing agent, is recommended (dose $1 \mathrm{mg} / \mathrm{kg}$ intravenously). Importantly, the duration of action of rocuronium is much longer than that of succinylcholine, lasting approximately $45 \min$ [15].

\section{Noninvasive Ventilation}

NIV, through use of either bilevel positive airway pressure (BPAP) or continuous positive airway pressure (CPAP), is both safe and effective in patients with cardiogenic 
pulmonary edema $[16 \bullet, 17]$. It improves both oxygenation and ventilation (with BPAP), reduces the work of breathing, improves symptoms, and has been associated with decreased intubation rates [16•]. Appropriate patient selection is critical. NIV is not a substitute for a definitive airway (i.e., endotracheal intubation). Patients with altered mental status or who are otherwise unable to protect their airway (e.g., handle secretions) should not receive NIV.

Robust data to support one method of NIV (BPAP vs CPAP) over another do not exist. Current American College of Emergency Physicians clinical practice guidelines support CPAP over BPAP on the basis of a single study suggesting an increased risk of myocardial infarction and also because of lack of robust differences in efficacy [17]. The Three Interventions in Cardiogenic Pulmonary Oedema (3CPO) Trialists found no mortality differences between CPAP, BPAP, and conventional oxygen therapy in ED patients with acute cardiogenic pulmonary edema [18]. They also found no differences between CPAP and BPAP in terms of intubation rates or mortality. NIV patients did have greater symptom improvement and less acidosis compared with patient who received traditional oxygen therapy [18].

We recommend the early use of NIV in appropriate patients with even moderate respiratory distress despite the $3 \mathrm{CPO}$ findings. Of note, there were no increases in the incidence of adverse events from NIV in the 3CPO trial. Its noninvasive nature, relatively easy applicability, improved symptoms, anecdotal and meta-analyses-reported reduction in intubation, and safety is why we continue to recommend NIV. CPAP may be started at $10-15 \mathrm{~cm} \mathrm{H}_{2} \mathrm{O}$ and titrated according to clinical status. Although arterial blood gas monitoring is not mandatory, for patients who fail to improve, a more nuanced understanding of the patient's respiratory status is helpful. A rising or high $\mathrm{CO}_{2}$ level may be an indication to add ventilatory support (i.e., BPAP if only CPAP was used), or if BPAP is already in place, it may be an indication to increase support or an indication of failure of NIV. Alternatively, continuous end tidal $\mathrm{CO}_{2}$ monitoring may also be used. Regardless, frequent reassessment of patients is necessary.

\section{Conclusion}

Patients with AHF present to the ED with shortness of breath and want to get better. Ascertaining the severity of dyspnea from the patients' perspective in addition to the physician's impression is important. Patients with severe respiratory distress may require immediate endotracheal intubation with RSI as the generally recommended approach. Some of these patients may also turn around rapidly with NIV, and this modality may be considered in appropriate patients. NIV is also recommended for patients with moderate to severe respiratory distress given its safety and efficacy profile.

Disclosure P. Pang: consultant for Astellas, Bayer, BGMedicine, EKR Therapeutics, J \& J, the Medicines Company, Medtronic, Novartis, Otsuka, Palatin Technologies, PDL BioPharma, Pericor Therapeutics, SigmaTau, Solvay Pharmaceuticals, SpringLeafTx, and Trevena; received honoraria from Alere, Beckman-Coulter, BiogenIdec, Corthera, Ikaria, Nile Therapeutics, Momentum Research, and Overcome; received research support from Abbott, Merck, and PDL BioPharma; received travel support from MyLife; received equipment support from Sonosite. M. Zaman: none.

\section{References}

Papers of particular interest, published recently, have been highlighted as:

- Of importance

•- Of major importance

1. • Mebazaa A, Pang PS, Tavares M, et al. The impact of early standard therapy on dyspnoea in patients with acute heart failure: the URGENT-dyspnoea study. Eur Heart J. 2010;31(7):832-41. This was one of the first articles to describe the impact of early treatment on dyspnea.

2. West RL, Hernandez AF, O'Connor CM, Starling RC, Califf RM. A review of dyspnea in acute heart failure syndromes. Am Heart J. 2010;160(2):209-14.

3. Metra M, Teerlink JR, Felker GM, et al. Dyspnoea and worsening heart failure in patients with acute heart failure: results from the Pre-RELAX-AHF study. Eur J Heart Fail. 2010;12(10):1130-9.

4. Stevenson LW, Hellkamp AS, Leier CV, et al. Changing preferences for survival after hospitalization with advanced heart failure. J Am Coll Cardiol. 2008;52(21):1702-8.

5. - Pang PS, Cleland JG, Teerlink JR, et al. A proposal to standardize dyspnoea measurement in clinical trials of acute heart failure syndromes: the need for a uniform approach. Eur Heart $J$. 2008;29(6):816-24. Despite the importance of dyspnea, how to measure it remains debated. This article proposes how to assess dyspnea in clinical trials, which also has relevance for clinical practice.

6. Teerlink JR, Metra M, Felker GM, et al. Relaxin for the treatment of patients with acute heart failure (Pre-RELAX-AHF): a multicentre, randomised, placebo-controlled, parallel-group, dosefinding phase IIb study. Lancet. 2009;373(9673):1429-39.

7. O'Connor CM, Starling RC, Hernandez AF, et al. Effect of nesiritide in patients with acute decompensated heart failure. N Engl J Med. 2011;365(1):32-43.

8. Gheorghiade M, Konstam MA, Burnett JC Jr, et al. Short-term clinical effects of tolvaptan, an oral vasopressin antagonist, in patients hospitalized for heart failure: the EVEREST clinical status trials. JAMA. 2007;297(12):1332-43.

9. McMurray JJV, Teerlink JR, Cotter G, et al. Effects of tezosentan on symptoms and clinical outcomes in patients with acute heart failure: the VERITAS randomized controlled trials. JAMA J Am Med Assoc. 2007;298(17):2009-19.

10. Committee on Health Care in America, Institute of Medicine. Crossing the quality chasm: a new health system for the $21 \mathrm{st}$ century. Washington: National Academies Press; 2001.

11. Metra M, Cleland JG, Davison Weatherley B, et al. Dyspnoea in patients with acute heart failure: an analysis of its clinical course, 
determinants, and relationship to 60-day outcomes in the PROTECT pilot study. Eur J Heart Fail. 2010;12:499-507.

12. Walls RM. Airway. In: Marx JA, Hockberger RS, Walls RM, et al., editors. Rosen's emergency medicine: concepts and clinical practice, vol. 1. 7th ed. Philadelphia: Mosby Elsevier; 2009.

13. McMurray JJ, Adamopoulos S, Anker SD, et al. ESC guidelines for the diagnosis and treatment of acute and chronic heart failure 2012: the Task Force for the Diagnosis and Treatment of Acute and Chronic Heart Failure 2012 of the European Society of Cardiology. Developed in collaboration with the Heart Failure Association (HFA) of the ESC. Eur Heart J. 2012;33(14):1787-847.

14. Weingart SD, Levitan RM. Preoxygenation and prevention of desaturation during emergency airway management. Ann Emerg Med. 2012;59(3):165.e1-75.e1.

15. Walls RM, editor. Manual of emergency airway management. 2nd ed. Philadelphia: Lippincott Williams and Wilkins; 2004.
16. - Vital FM, Saconato H, Ladeira MT, et al. Non-invasive positive pressure ventilation (CPAP or bilevel NPPV) for cardiogenic pulmonary edema. Cochrane Database Syst Rev. 2008(3): CD005351. This review describes the value of NIV, and includes the article by Gray et al. in its analysis. Overall, NIV continues to have value.

17. Silvers SM, Howell JM, Kosowsky JM, Rokos IC, Jagoda AS. Clinical policy: critical issues in the evaluation and management of adult patients presenting to the emergency department with acute heart failure syndromes. Ann Emerg Med. 2007;49(5): 627-69.

18. Gray A, Goodacre S, Newby DE, Masson M, Sampson F, Nicholl J. Noninvasive ventilation in acute cardiogenic pulmonary edema. N Engl J Med. 2008;359(2):142-51. 